Disponible on-line en:

http://revistalogopedia.uclm.es

Revista de Investigación en Logopedia 4 (2014) 1-27.

ISSN-2174-5218

\title{
Evaluación del componente pragmático en el Síndrome de Down a través del Protocolo Rápido de Evaluación Pragmática
}

\author{
Esther Moreno y Félix Díaz \\ Universidad de Castilla la Mancha, España
}

\begin{abstract}
Resumen
La bibliografía sobre el componente pragmático en personas con Síndrome de Down (SD) ofrece resultados difusos. No hay consenso en torno a dos cuestiones fundamentales: (1) si el componente pragmático es un punto fuerte en estas personas y (2) en qué habilidades pragmáticas presentan un buen rendimiento. Los objetivos de esta investigación han sido: (1) el análisis de las capacidades pragmáticas de un sujeto con SD de alto rendimiento y (2) la comprobación de la utilidad de un instrumento de evaluación concreto para llevar a cabo este análisis en este colectivo. La recogida de información se ha realizado por medio de grabaciones de muestras de lenguaje obtenidas en diferentes situaciones de interacción con distintos interlocutores, en el contexto de la Asociación a la que acude el sujeto. Para el análisis de las muestras hemos empleado la estructura y clasificación del Protocolo Rápido de Evaluación Pragmática (PREP-INIA) propuesto por Gallardo et al. (2008), que valoramos por su aplicabilidad, sistematicidad y claridad. Los resultados de esta investigación aportan información cualitativa sobre el componente pragmático en el caso seleccionado de cara a establecer líneas generales de acción. Estos resultados constituyen un primer paso hacia un estudio más exhaustivo del componente pragmático en este colectivo con la finalidad de diseñar instrumentos de evaluación y protocolos de intervención más eficaces.

Palabras clave: Pragmática; PREP; Síndrome de Down.
\end{abstract}

\section{Assessment of the pragmatic component in Down's syndrome through the Quick Pragmatic Assessment Protocol}

\begin{abstract}
The literature on the pragmatic component in persons with Down Syndrome (DS) shows no consensus on whether the pragmatic component is a strong asset in these persons or what pragmatic abilities or functions render good performance. The aims of this research have been to (1) analyze the pragmatic features of a person with high-performance DS, and (2) test the usefulness of a specific assessment instrument for this component in this collective. The information was collected by recording language samples in various interaction situations with different conversation partners, in the context of the Association he attends. To analyze the samples we have followed the structure and classification of the Quick Pragmatic Assessment Protocol (Gallardo et al., 2008), which we value for its ease of use, systematicity and clarity. The results of this research provide qualitative information on the pragmatic component in the selected case towards planning general treatment lines. These results constitute a first
\end{abstract}


step towards a more exhaustive study of the pragmatic component in this collective in order to devise more efficient assessment instruments and intervention protocols.

Key words: Down syndrome; Pragmatics; QPPA.

Correspondencia con los autores: felix.diaz@uclm.es

Recibido 15 de septiembre. Primera revisión 15 de noviembre. Aceptado 18 de diciembre de 2013.

Agradecimientos: Este trabajo ha sido desarrollado al amparo del Proyecto de Investigación con referencia FFI2012-39325-C03-01del Ministerio de Economía y Competitividad de España.

Agradecemos la inestimable colaboración de F, V, D, sus familias, Noelia Núñez Andrés (logopeda de la Asociación Síndrome de Down de Toledo) y ASDOWNTO como entidad. Agradecemos también las exhaustivas anotaciones y comentarios de uno de los revisores.

\section{Introducción}

El síndrome de Down (SD) fue descrito inicialmente por John Langdon Down (1866), quien trató de establecer las causas y características físicas comunes de lo que llamó "the Mongolian type of idiocy". Posteriormente, el investigador y genetista Jérôme Lejeune (1963) descubrió que este síndrome se debía a una alteración genética producida por la presencia de una copia extra en el par 21 de cromosomas (trisomía 21). Entre los síndromes genéticos que cursan con discapacidad intelectual, el SD es actualmente uno de los más conocidos. Se caracteriza por la presencia de determinados rasgos físicos, retraso del desarrollo y distintos grados de discapacidad intelectual. En relación con el desarrollo del lenguaje, muchos niños con SD muestran dificultades durante la adquisición de las primeras palabras. Su vocabulario crece más lentamente que el de los niños con desarrollo típico y, aunque son capaces de construir estructuras de dos palabras, tienen dificultades para aprender las reglas habladas y las formas gramaticales correctas de las oraciones (Rondal, 1988; Miller, 1988).

En el ámbito fonológico, Roberts, Long, Malkin, Barnes, Skinner y Hennon (2005) encontraron una tendencia a la omisión de sílabas, reducción de grupos consonánticos y omisión de consonantes en los niños con SD. Por lo que se refiere al ámbito léxico, su nivel de comprensión es similar al de niños de desarrollo típico de edades mentales similares (Laws \& Bishop, 2003), pero el crecimiento temprano de su vocabulario expresivo es más lento (Berglund, Eriksson \& Johansson, 2001). Presentan longitudes de emisión (LMEV) inferiores a las de los niños con desarrollo típico (Chapman, Seaung, Schwartz \& Kay-Raining, 1998); dificultad con la producción de morfemas gramaticales (Berglund et al., 2001); y producción reducida de verbos auxiliares (Hesketh \& Chapman, 1998).

Hasta el momento, el estudio de las habilidades pragmáticas en SD no ha seguido criterios homogéneos (Johnston \& Stanfield, 1997). La revisión bibliográfica 
arroja afirmaciones de carácter vago y general. Así, Abbeduto, Warren y Conners (2007) y Roberts et al. (2007) coinciden en defender que las personas con SD presentan un "perfil de habilidades pragmáticas" con puntos fuertes y débiles.

A continuación, ofrecemos una revisión del estado de la cuestión en torno al componente pragmático en SD, estructurada en tres apartados: (1) estudios sobre primeros momentos de la adquisición del lenguaje, (2) estudios sobre edades posteriores y (3) una clasificación estructurada de hallazgos específicos.

En las etapas más tempranas de adquisición, Greenwald y Leonard (1979) mostraron que las intenciones comunicativas de los niños con SD estaban peor desarrolladas que las de niños con desarrollo típico, ya que vocalizaban menos que los niños con desarrollo típico para propósitos socio-comunicativos. Años más tarde y en contraste con estudios anteriores, Coggins, Carpenter y Owings (1983) no encontraron diferencias significativas entre grupos en cuanto a intenciones comunicativas durante la interacción. Wootton (1990) analizó los componentes verbales y no verbales que se ponían en juego en situaciones de interacción entre madres y niños con SD durante una tarea de lectura de cuentos y concluyó que usaban el gesto de señalar para iniciar la interacción y que la adquisición de este gesto se prolongaba en el tiempo. Beeghly, Weiss-Perry y Ciccheti (1990) compararon a niños con deficiencia mental y de desarrollo típico durante una tarea similar y concluyeron que los niños con SD empleaban menos respuestas que los de desarrollo típico, pero no diferían en el mantenimiento del tema. En conjunto, estas investigaciones parecen sugerir que los niños con SD tienen intención comunicativa y la manifiestan desde los primeros años mediante vocalizaciones y gestos.

En edades más avanzadas, los hallazgos sugieren que la competencia comunicativa de estos niños está intacta a pesar de sus déficits (Price-Williams \& Sabsay, 1979; Leudar, Fraser \& Jeeves, 1981). Igualmente, en el estudio de Nisbet, Zanella y Miller (1984) los sujetos demostraron una competencia lingüística avanzada. Asumían roles de habla más competentes y ajustaban su capacidad de lenguaje para tomar las riendas de la conversación, haciendo más preguntas, introduciendo más temas y usando expresiones más largas que sus iguales de desarrollo típico. Beeghly, WeissPerry y Cicchetti (1990) demostraron que las habilidades discursivas de las personas con SD no estaban alteradas y realizaban la misma variedad de funciones comunicativas que sus iguales de desarrollo típico. Roberts, Price y Malkin (2007) apoyaron estos resultados, pero además demostraron que la calidad del mantenimiento del tema puede 
ser diferente entre grupos. Los niños con desarrollo típico emplean más formas elaborativas del mantenimiento del tema que los niños con SD, quienes empleaban formas más simples en adecuación y calidad.

En síntesis, las habilidades comunicativas de las personas con SD no parecen ser un gran hándicap. Se desenvuelven correctamente durante una conversación y afrontan sus demandas (Rondal y Comblain, 1996), a pesar de presentar problemas en algunos aspectos concretos.

Los estudios revisados hasta aquí hacen referencia a categorías y sistemas de clasificación de la pragmática poco homogéneos. Esto es comprensible, dada la ausencia de estándares claros para la descripción de la competencia pragmática. En esta investigación hemos utilizado una clasificación estructurada, aportada por Gallardo (2009), que divide la pragmática en tres categorías: enunciativa, textual e interactiva. Esta clasificación resulta compatible con algunos estudios sobre desarrollo de la pragmática en SD, que revisamos a continuación.

-La pragmática enunciativa (Gallardo, 2009) engloba aquellas categorías que surgen de la consideración de cada enunciado como una acción intencional de un hablante. En esta categoría encontramos los actos de habla y los significados inferenciales.

Encontramos estudios que se ocupan de los actos de habla indirectos. Abbeduto y Rosenberg (1980) encontraron que sus participantes eran capaces de producir actos de habla indirectos, concretamente responder a preguntas con las respuestas requeridas. Sin embargo, Owens y MacDonald (1982) compararon los actos indirectos en niños con SD y niños de desarrollo típico y concluyeron que algunos actos de habla diferenciaban a los dos grupos, como la dificultad para realizar preguntas pertinentes.

Años más tarde, Abbeduto, Davies y Furman (1988) afirmaron que, si bien las personas con discapacidad intelectual presentan un retraso en la adquisición de la comprensión de peticiones indirectas "convencionales", su desarrollo sigue las pautas del desarrollo normal. Sin embargo, Abbeduto, Davies, Solesby y Furman (1991) señalaron la posibilidad de que existan alteraciones en la comprensión de enunciados indirectos que comunican significados implícitos no convencionales, como las sugerencias o las ironías.

En síntesis, si bien estas personas pueden producir actos de habla indirectos, éstos son diferentes a los de niños con desarrollo típico y pueden presentar dificultades a la hora de interpretar actos indirectos de mayor complejidad. 
-La pragmática textual (Gallardo, 2009) engloba los aspectos que dotan de coherencia y cohesión al mensaje.

Beeghly et al. (1990), en un estudio con 17 niños y 11 niñas con SD, encontraron que éstos no diferían de los niños de edad evolutiva similar en su capacidad para mantener temas. En esta línea, Cicchetti y Beeghly (1990) compararon a niños diagnosticados de SD con niños sin SD de equivalente Longitud Media de Emisión Verbal (LMEV). Comprobaron que los niños con SD exhibían mayor habilidad en mantenimiento temático así como en el empleo de las reglas de pertinencia conversacional.

Boudreau y Chapman (2000) y Miles y Chapman (2002) estudiaron las habilidades narrativas de personas con SD, sugiriendo que la comunicación de contenidos narrativos puede ser una relativa fortaleza en ellos si usan apoyos visuales. Niños, adolescentes y jóvenes con SD incluían un número similar de elementos narrativos que niños sin diagnóstico de equivalente edad mental al relatar una película muda. Más aún, los jóvenes con SD recordaban más elementos narrativos y referencias temáticas que los niños sin diagnóstico. Sin embargo, los jóvenes con SD usaban menos recursos de cohesión que los niños sin diagnóstico de equivalente edad mental (pero se igualaban con los niños de equivalente edad de lenguaje). En la misma línea, Abbeduto (2006) sugirió que, en tareas no presenciales de re-narración de fragmentos de una novela, los niños con SD expresaban mensajes menos claros que los niños con desarrollo típico.

-La pragmática interactiva (Gallardo, 2009) engloba aspectos interactivos de la comunicación, como son la toma y agilidad de turnos, la predictibilidad y prioridad de las intervenciones del sujeto o su relativa participación en la conversación.

Coggins y Stoel-Gammon (1982), en un estudio con cuatro sujetos con SD, encontraron que eran sensibles a las demandas de aclaración de sus interlocutores. Los sujetos reparaban todas las rupturas conversacionales cuando su interlocutor solicitaba respuestas de aclaración.

Sin embargo, recientemente Abbeduto, Murphy, Kover, Karadottir, Amman y Bruno (2008) encontraron que los adolescentes con SD señalaban menos la ausencia de comprensión de los enunciados, durante una tarea que requería que solicitaran peticiones de aclaración o información adicional. Estos resultados sugieren que, si bien las personas con SD son sensibles y responden a las demandas de aclaración por parte 
de sus interlocutores, señalan menos la ausencia de comprensión de enunciados cuando son ellos los que requieren información adicional sobre un tema concreto.

Peskett y Wootton (1985) examinaron los solapamientos producidos en conversaciones de niños con SD de 3 y 4 años de edad. Sugirieron que los sujetos desplegaban estrategias individualizadas para remediar las situaciones que se crean cuando hay solapamiento.

Aunque estos resultados indican que estas personas no son igual de competentes en todas las habilidades pragmáticas, las investigaciones coinciden en que, por lo general, cuentan con una buena competencia comunicativa y pueden compensar sus déficits usando estrategias específicas cuando la situación lo requiere.

En síntesis, nuestra revisión pone de manifiesto dos problemas metodológicos en la investigación sobre pragmática en SD. Por una parte, la mayoría de las investigaciones se apoyan en tareas relativamente específicas, diseñadas ad hoc para responder cuestiones muy concretas sobre capacidades previamente determinadas. Por otra parte, esta especificidad, unida a la ausencia de una teoría sistemática sobre el déficit pragmático en general, dificulta la opción de integrar distintos resultados para construir una descripción global, organizada y a la vez detallada de la competencia pragmática en SD.

Nuestra propuesta está dirigida a resolver estos problemas, (1) partiendo de un muestreo de situaciones de habla variadas y representativas, donde el sujeto pueda desplegar toda su competencia pragmática; y (2) apoyándonos en una clasificación sistemática de las diferentes habilidades específicas que componen la competencia pragmática, como es la citada clasificación de Gallardo (2009).

A continuación ofrecemos una valoración cualitativa de las habilidades pragmáticas de una persona con SD, utilizando el PREP (Gallardo, 2008, 2009; ver Anexo I). El objetivo principal de esta valoración es poner a prueba la capacidad descriptiva de este instrumento de evaluación para caracterizar el componente pragmático en SD.

\section{Método}

Realizamos un análisis cualitativo del componente pragmático a partir de muestras de lenguaje en un caso único de SD de alto espectro, mediante la aplicación del PREP. 


\section{Participantes}

El participante es FCG, varón de 17 años que acude a sesiones de logopedia en una Asociación de SD en España. FCG tiene un alto rendimiento pragmático, puede mantener una conversación más o menos fluida y es amable al trato, lo cual permite evaluar su competencia pragmática cubriendo la totalidad de ítems del PREP-INIA. A los 8 años comenzó a acudir a la Asociación de Down en la que participa hoy. Su escolarización se ha realizado siempre en colegios ordinarios. No ha sido sometido a pruebas estandarizadas de evaluación del lenguaje.

\section{Materiales}

El Protocolo Rápido de Evaluación Pragmática (PREP-INIA; Gallardo, 2008) surge como simplificación del perfil PerLA de Evaluación pragmática desarrollado por Gallardo (2006). Está diseñado como un instrumento de aplicación sencilla, eficiente en términos de disponibilidad temporal en el ámbito clínico. Sus autoras lo describen como sencillo de interpretar, proporcionando al clínico un primer acercamiento al déficit pragmático que permitiría después una exploración más detallada de las habilidades afectadas. El PREP se compone de ítems explicados y divididos según la clasificación de tipos de pragmática antes comentada.

El diseño del PREP permite recorrer una muestra de lenguaje suficientemente amplia y valorar a un sujeto en cada una de sus categorías. El instrumento no especifica el procedimiento para obtener los datos y ni siquiera impone necesariamente la necesidad de una muestra transcrita, pudiéndose valorar las categorías a partir de la observación directa o confiando en la memoria y capacidad de observación del clínico en su relación habitual con el sujeto.

\section{Muestra}

La primera autora de este trabajo realizó videograbaciones en varias situaciones comunicativas y con diferentes interlocutores, siempre en el contexto de la Asociación a la que acude el participante. Obtuvimos previamente el consentimiento informado de todos los participantes en las grabaciones. Los datos de los diferentes registros se detallan en la Tabla 1. 
Tabla 1. Datos de los diferentes registros

\begin{tabular}{|c|c|c|c|}
\hline$\underline{\text { Registro }}$ & $\underline{\text { Actividad }}$ & Duración $^{\prime}$ & Participantes $^{2}$ \\
\hline $\begin{array}{l}\text { Entrevista con su } \\
\text { tutora }\end{array}$ & $\begin{array}{l}\text { Diálogo. Presentación y } \\
\text { hobbies. }\end{array}$ & $\begin{array}{l}\text { 04' 57”' } \\
\text { (Vídeo 1) }\end{array}$ & $\begin{array}{ll}\text { - } & \text { Sujeto (F.) } \\
\text { - } & \text { Tutora (T.) }\end{array}$ \\
\hline $\begin{array}{l}\text { Sesión de Logopedia } \\
\text {-Conversación guiada }\end{array}$ & $\begin{array}{l}\text { Conversación espontánea y } \\
\text { discusión de la tarea } \\
\text { propuesta }\end{array}$ & $04{ }^{\prime} 42^{\prime}$ & $\begin{array}{ll}- & \text { Sujeto (F.) } \\
\text { - } & \text { Compañera (D.) } \\
\text { - } & \text { Logopeda (N.) }\end{array}$ \\
\hline $\begin{array}{l}\text {-Reconocimiento } \\
\text { de famosos }\end{array}$ & Resolución de una tarea. & $\begin{array}{l}\text { Registro } 1 \\
08 \text { '02" } \\
\text { Registro } 2 \\
08 \text { '37'" }\end{array}$ & $\begin{array}{ll}- & \text { Sujeto (F.) } \\
\text { - } & \text { Compañera (D.) } \\
\text { - } & \text { Logopeda (N.) }\end{array}$ \\
\hline Monólogo & $\begin{array}{l}\text { Monólogo sobre lo que sabe } \\
\text { del síndrome de Down }\end{array}$ & $\begin{array}{l}\text { 04:56" } \\
\text { (Vídeo 2) }\end{array}$ & - $\quad$ Sujeto (F.) \\
\hline $\begin{array}{l}\text { Conversación } \\
\text { pareja }\end{array}$ & $\begin{array}{l}\text { Hablan sobre sus estudios, } \\
\text { sobre un incidente concreto } \\
\text { y sobre su relación. }\end{array}$ & $\begin{array}{l}\text { Registro } 1 \\
\text { (01'03") } \\
\text { Registro } 2 \\
\text { (05'46”) } \\
\text { (Vídeo 3) }\end{array}$ & $\begin{array}{ll}\text { - } & \text { Sujeto (F.) } \\
\text { - } & \text { Compañera (D.) }\end{array}$ \\
\hline $\begin{array}{l}\text { Conversación con } \\
\text { varios interlocutores }\end{array}$ & $\begin{array}{l}\text { Conversación sobre el } \\
\text { deporte, la amistad entre } \\
\text { ellos, la asociación... }\end{array}$ & $\begin{array}{l}\text { Registro } 1 \\
\text { (04'56") } \\
\text { Registro } 2 \\
\text { (10'14") } \\
\text { (Vídeo 4) } \\
\text { Registro } 3 \\
\text { (01'58") }\end{array}$ & $\begin{array}{ll}\text { - } & \text { Sujeto (F.) } \\
\text { - } & \text { Compañera (D.) } \\
\text { - } & \text { Compañero (V.) }\end{array}$ \\
\hline
\end{tabular}

\section{Resultados}

En este apartado nos detendremos en cada categoría evaluada por el PREP. Ilustraremos cada ítem con fragmentos de muestra y justificaremos aquellos casos que no han sido evaluados. Nuestro análisis, por tanto, consistirá en un recorrido por las categorías de

\footnotetext{
${ }^{1}$ Los registros cuya duración está resaltada en negrita son los que se han utilizado para ilustrar los ítems del PREP. A lo largo del análisis, citaremos fragmentos de estos registros haciendo referencia al número de vídeo indicado en esta columna.

${ }^{2}$ D y V, mencionados en esta columna como "compañeros", son usuarios de la Asociación con SD; D es mujer de 17 años y $\mathrm{V}$ es varón de 18 años.
} 
las tres pragmáticas de Gallardo (2009), deteniéndonos en cada ítem del PREP para ilustrarlo sobre fragmentos de transcripción del caso. Algunos ítems no han sido evaluados y discutiremos específicamente por qué.

\section{Pragmática enunciativa}

1. Actos de habla: El acto de habla es la unidad básica de comunicación linguíistica con la que se realiza una acción; el concepto fue utilizado inicialmente por Austin (1962) y consolidado por otros autores como Searle (1969). En el PREP, las cinco categorías relativas a actos de habla se relacionan con las dimensiones locutiva e ilocutiva.

Dimensiones de los actos de habla:

\subsection{Articulación y modulación sonora}

Vídeo $1^{3}$. (00’00”-00’22”)

- Tutora: Ehh / pues empieza diciendo tu nombre, hola me llamo F

- F: Me llamo, me llamo-, me llamo F, tengo 17 años

- Tutora: ¿Dónde vives F?

- $\quad$ F: En Peraleda

- Tutora: Y cómo/ ¿pero te sabes la dirección em justa de tu casa, la calle?

- F: Peraleda, calle ale- ale- ale-gría cuarenta y ocho, cuarenta y ocho.

En este fragmento se aprecian dificultades articulatorias en algunas palabras. No obstante, en este registro la interacción está muy dirigida por la interlocutora y el sujeto puede estar fallando por el estrés añadido que supone este formato dirigido. Pero estos fallos están presentes también en situaciones de conversación más espontánea.

\subsection{Acceso Léxico}

Vídeo 4 (02’07’)

- F: ya/Yo lo que pienso de- de- de- del deporte olimpiamente que el deporte es un juego lim- bueno/ a partir de ya un juego limpio donde se practica las piernas en natación, las piernas la espalda (...)

\footnotetext{
${ }^{3}$ Las convenciones de transcripción se recogen en el Anexo 2.
} 
Apreciamos un uso inadecuado del repertorio léxico manifestado en la expresión "a partir". La dificultad puede deberse a desconocimiento del uso de la partícula. A pesar de este fallo, en el conjunto de la muestra apenas hay alteraciones del acceso léxico.

\subsection{Manejo de pausas y silencios}

En ocasiones se producen silencios incómodos durante la conversación, como en el fragmento que presentamos a continuación, tomado del vídeo 4, minuto (00'27”), donde $\mathrm{F}$, respondiendo a una pregunta de $\mathrm{D}$, se interrumpe repentinamente, queda en silencio y cambia de tema bruscamente:

- D: (...) ¿Qué tocas de piano?

- F: pues / lo q- lo q- lo que toco es do, re, mi, fa, sol, la, si do, do / con las dos manos así / y (4) (coloca las manos y mueve los dedos para simular cómo toca) yo lo que pienso, la verdad en el deporte que el deporte es un juego limpio donde se puede practicar en muchas cosas ¿sabes?

El silencio dura 4 segundos, pero no llega a perturbar el flujo conversacional. Por una parte, está parcialmente cubierto por una acción no verbal que explica la anterior emisión; a la vez, el silencio parece preparar el cambio de tema (indicando una continuación del turno con el copulativo “y”), de manera que la espera es proyectada y F introduce el tema nuevo sin gran dificultad.

Sin embargo, en otras situaciones el silencio se emplea adecuadamente como una estrategia, como por ejemplo al finalizar la exposición del monólogo. Cuando F no tiene más información que aportar, cierra el monólogo y, al ver que le siguen grabando, permanece callado e insiste en cerrar el tema.

2. Actos ilocutivos. Las emisiones demuestran que F tiene una comprensión suficiente de lo que se le dice y son las adecuadas para conseguir su propósito comunicativo, aunque puedan tener fallos gramaticales o léxicos

Por lo general, F sí muestra comprensión de lo que se le dice, tanto cuando se le hacen preguntas concretas (formato entrevista) como en conversación con varios 
interlocutores. Así se aprecia en el fragmento del Vídeo 1 (00'11”) citado más arriba. He aquí otro ejemplo:

Vídeo 4 (03'52”)

- D: A veces F a mí la piscina cuando tú te tiras allí/es que me da per- pereza

- F: ¿Te da pereza o te da miedo?

- D: me da miedo

- F: pues tienes que vencer a- al miedo, porque yo- yo,

\section{$\underline{\text { Tareas de edición }}$}

3. Ilocutividad. Pese a tener problemas léxicos o articulación consigue transmitir su intención comunicativa con:

1.1 Exclamaciones, entonaciones marcadas

1.2 Gestos y actos no verbales

1.3 Circunloquios, rodeos...

Puesto que $\mathrm{F}$ no presenta problemas de léxico o articulación que requieran ser compensados a través de estos recursos, estos ítems no son evaluables. F emplea exclamaciones y entonaciones marcadas a lo largo del discurso para hacerse entender en cada momento y la entonación es adecuada para cada acto comunicativo en su contexto secuencial; pero en ningún momento necesita alterar el tono o el volumen para compensar dificultades léxicas o articulatorias. De manera similar, aunque gestos y actos no verbales acompañan el lenguaje del sujeto en diferentes interacciones comunicativas, no son sustitutorios ni compensatorios. Y tampoco necesita acudir a circunloquios para compensar dificultades de acceso léxico o articulación; si bien comentaremos posteriormente el uso de circunloquios en relación con aspectos de coherencia y gestión temática.

4. F muestra conciencia de su propia dificultad iniciando él mismo rectificaciones en los puntos problemáticos.

F realiza auto-reparaciones conversacionales donde presenta problemas de articulación; repite la palabra hasta que la produce completa. No se han apreciado autorectificaciones conversacionales en los registros que hemos seleccionado para el análisis. 


\section{$\underline{\text { Inferencias }}$}

5. Inferencias tópicas lexicalizadas. Al plantearle emisiones que contienen frases hechas, refranes, etc. de sentido figurado, demuestra haberlas entendido correctamente o las usa él mismo. Este elemento no se ha podido evaluar, ya que la muestra no contenía ninguna mención de este tipo por F o sus interlocutores.

6. Implicaturas conversacionales. Su conversación (pese a posibles fallos léxicos o articulatorios) avanza con relativa fluidez, respetando las máximas conversacionales de cooperación que exigen proporcionar información:

\subsection{Veraz (Máxima de cualidad)}

Como criterio de verdad para la máxima de cualidad usamos la confirmación de la información ofrecida por F por su logopeda. En ese sentido, su discurso se ajustó a la realidad.

\subsection{Suficiente, ni escasa ni excesiva (máxima de cantidad)}

Vídeo 4 (03'19”)

- D: A veces F a mí la piscina cuando tú te tiras allí/es que me da per- pereza

- F: ¿Te da pereza o te da miedo?

- D: me da miedo

- F: pues tienes que vencer a- al miedo, porque yo- yo

- D: es que si yo/yo me rompo las piernas

- F: Tú te rompes las piernas/pues ¡yo me tiro y no me rompo las piernas!

- F: si tú te tiras y lo mejo- que hay cuando tú vas a una competición y te titiras y pareces una sirena y puedes ganar/si no te tiras pues lo u- lo u- lo único que haces es perder tiempo es perder tiempo en natación/ y yo digo igual que en que en el baloncesto si el árbitro pita por posesión posesión tenemos que ir al entrenador, a donde está el entrenador, el entrenador nos dará las instrucciones tú tienes que ir de defensa, tú tienes que ir de base nosotros nos ponemos nos colocamos así (levanta los brazos) como los grandes jugadores y defendemos. 
En este fragmento, $\mathrm{F}$ aporta más información que la requerida por el interlocutor; está hablando con D sobre la natación y tirarse o no del trampolín y de repente cambia de tema hacia el baloncesto haciendo aportaciones que no son relevantes y que resultan excesivas para este intercambio.

Sin embargo, en situaciones con mayor control externo, como es la entrevista, el sujeto muestra una alteración de la máxima de cantidad en el sentido contrario: responde a las preguntas de manera muy breve y sin aportar toda la información requerida. La parquedad de las respuestas de $\mathrm{F}$ hace que la tutora tenga que plantearle preguntas para que aporte más información ${ }^{4}$.

6.3 Clara, concreta (Máxima de manera)

1.4 Vídeo 4 (08'05”)

- F: Yo lo que pienso y le digo un mensaje a mi entrenador favorito que se llama Jose, que es el mejor entrenador que tenemos de la Asociación él nos entrena y él nos da las instrucciones que tenemos que hacer, porque por ejemplo el otro día le puse un wa- un wasap diciéndole que estaba viendo el deporte, el baloncesto, por ejemplo el Real Madrid y o el los ang- los ángeles Lakers/esos ganan ¿Por qué? porque mayores.

La exposición parece clara en un inicio pero se desvía y es difícil entender lo que F quiere decir, pues pasa de hablar de su entrenador a hablar de equipos como el Real Madrid y Los Ángeles Lakers. Se pierde la máxima de manera por exceso de derivación temática y breves descarrilamientos temáticos.

\footnotetext{
${ }^{4}$ No descartamos que esta parquedad esté asociada a lo que Díaz (2010) ha considerado el 'paradigma de respuesta mínima', un rasgo institucional de la interacción conversacional en la clínica logopédica por el cual los pacientes interrogados por logopedas proporcionan sistemáticamente la mínima información lógica requerida.
} 
6.4 Relevante (Máxima de pertinencia)

Vídeo 3 (conversación en pareja) (01'40”)

- F: yo te voy a decir una cosa que tienes que saber y que lo sepa Miguel también.

- D: vale.

- F: Mañana ma- ma- mañana/¿mañana lo vas a ver?

- D: Mañana sí porque me toca con él mañana/ en la clase.

- F: una cosa ¿quie- quier- que te dé un men- um men- un mensajito?

- D: Vale.

- F: le vas a decir ¿eh? lo que te he dicho lo de que cuando te mareaste y eso/¿también estuvo?

- D: él lo sabe ya

- F: ¿también estuvo Miguel?

- D: Él lo sabe ya/Miguel sobre eso

- F: pero ¿que también estuvo cuando te mareaste?

- D: No porque él estuvo en la calle, fumando.

- F: Ah, y/bueno y sé bueno sé/que Javi el de religión sabe que/que aunque yo no esté allí pero me gustaría decirle quee que también es un hombre como yo y que te cuide mucho/ (...)

En este fragmento apreciamos una violación de la máxima de manera y de pertinencia: en el inicio del fragmento, F propone a D mandar un mensaje a Miguel, que es el profesor de Matemáticas de D. De manera que, en un principio, parece focalizar la conversación hacia ese propósito. Pero no llega a precisar la propuesta, pasando a hablar del profesor de religión.

\subsection{Implicaturas anómalas. En ocasiones, comprendida a partir de sentidos figurados.}

En los registros evaluados, no se han encontrado situaciones en las que haya que hacer inferencias a partir de la situación, por lo cual este ítem no ha sido evaluado. No obstante, hemos de resaltar la importancia de un hecho que nos ha llamado especialmente la atención y es que a menudo el sujeto entiende las preguntas de manera muy literal. Así lo podemos observar en este fragmento: 
Vídeo 1; minuto (00'25")

- $\quad \mathrm{T}:$ Amm y/¿estás estudiando?

- F: Sí.

- T: ¿Dónde estudias?

- F: En el San Marcos

- T: ¿Y qué estás estudiando?

- F: Estoy estudiandooo, lengua, matemáticas eh ética/

- T: pero/¿En qué curso estás?

- F: estoy en cuarto

- T: En cuarto de la ESO

- F: Sí

\section{Pragmática textual}

7. Coherencia: Al construir un relato o explicar una idea respeta las estructuras lógicas en el orden en el que cuenta las cosas, la manera en que relaciona las ideas, la selección de información necesaria...

Exceptuando el cambio de tema del principio, ya comentado anteriormente, $\mathrm{F}$ respeta el tema que se está tratando y lo preserva desde el principio hasta el final del intercambio. Sin embargo, la alternancia de referencias a distintos subtemas genera confusión y falta de coherencia, es decir, aporta información excesiva y mal organizada en la progresión del intercambio. 
Vídeo 4 (00'37”- 01:30”)

- F: pues/lo q- lo q- lo que toco es do re mi fa sol la si do, do/con las dos manos así/y/yo lo que pienso, la verdad en el deporte que el deporte es un juego limpio donde se puede practicar en muchas cosas ¿sabes?

- D: ya

- F: por ejemplo en natación, hay que saber bien la técnica de natación

- D: Sí.

- F: porque si no nn- no ganamos, si no no ganamos, igual pienso de b- b- de baloncesto, si cuando estamos entrenando sabemos entrenar y cuando llegamos al partido de Albace- tío, no sabemos jugar. ¿o no te acuerdas en lo cuando fuimos al Albacete?

- D: yo también me acuerdo de Alba- Alba- Albacete.

- F: No pero yo no digo de na- na- natación yo digo de baloncesto.

- D: Ah, vale.

- F: yo lo que pienso tío es, que grandes jugadores sean como nosotros eh? por ejemplo, Kobi Bryan el de Los Ang- Angeles Lakers, eso tiene un récord (Mueve la mano de un lado a otro para indicar mucho)

8. Su discurso se ajusta al tema que se está tratando y cuando cambia de tema lo hace de manera fluida, sin brusquedad ni rupturas temáticas evidentes.

El ejemplo tomado del Vídeo 4, minuto (00'27”), mostrado más arriba, ilustraba una peculiar transición temática de "clases de piano" a "el deporte es un juego limpio". Normalmente, y al ser él quien dirige los intercambios comunicativos en conversaciones no guiadas por profesionales, F orienta las conversaciones hacia temas que conoce, como el deporte. Los cambios de tema se acentúan cuando se habla de algo que él no domina, por ejemplo las clases de piano, como vimos en aquel fragmento, donde contesta escuetamente a la pregunta de $\mathrm{D}$ y pasa rápidamente a hablar del deporte.

9. Cohesión. Tiene la suficiente capacidad léxica como para usar diversas maneras de referirse a lo mismo, en lugar de repetir siempre las mismas palabras o dejar la frase incompleta... 
En el monólogo, por ejemplo en el fragmento del Vídeo 2_(02'32") mostrado más arriba, muchos enunciados se quedan a medias y la continuidad de referencia a un objeto usando la misma expresión contribuye al incumplimiento de la máxima de manera.

10. Su construcción de las palabras es completa, utilizando adecuadamente las terminaciones de sustantivos y de verbos, respetando las concordancias de tiempo, género, número, así como los artículos.

En este sentido el sujeto no muestra dificultades y es capaz de utilizar la morfología adecuada en todos los intercambios.

11. Su construcción de las frases y oraciones es convencional y utiliza enunciados de estructura completa, tanto en las oraciones (sujeto+predicado) como en constituyentes menores (preposición+término auxiliar+verbo, etc.)

Estructura las oraciones de manera adecuada respetando y siguiendo la estructura convencional de sujeto/verbo/complemento.

\section{Pragmática interactiva}

12. Su participación se realiza con la agilidad y rapidez propias de la conversación cotidiana, sin lentitud ni aceleración exagerada (Agilidad del turno)

Hemos visto en diversos fragmentos del Vídeo 4 cómo, en conversaciones con iguales, $\mathrm{F}$ frecuentemente asigna siguientes turnos y dirige la conversación hacia el tema del deporte. Dado que sus interlocutores tienen un menor nivel de ejecución verbal, le permiten llevar la voz cantante y hacer preguntas para incorporar a los demás miembros de la conversación. Por lo general la gestión de turno es adecuada ya que se realiza con agilidad y sin solapamiento entre miembros.

13. Aparentemente, su nivel de participación activa en la conversación (cantidad de turnos y palabras) es proporcional al de los otros participantes; coopera bien en el desarrollo de la toma de turno y respeta la alternancia

F coopera bien en la toma de turno pero en ocasiones no respeta la alternancia, como en el fragmento del Vídeo 4, (03'19”) mostrado más arriba, donde D está iniciando una descripción de su experiencia con la educación física y, antes de que llegue a exponerla, 
$\mathrm{F}$ vuelve a tomar el turno, interrumpiendo. Como hemos comentado, en las conversaciones con iguales $\mathrm{F}$ dirige la interacción, establece turnos, introduce temas y se mantiene siempre activo.

14. Utiliza igualmente turnos de respuesta a preguntas ajenas, como turnos que informan o preguntan (predictibilidad: tipos de intervención).

$\mathrm{F}$ responde a preguntas formuladas directamente, como las que hemos visto en fragmentos tomados de la entrevista y cuando se refieren a él en una conversación cotidiana, como por ejemplo, en el minuto 00:17 (donde responde a una pregunta concreta) y en el 02:42" (donde realiza una pregunta a un interlocutor y toma la palabra para informarle de algo) del vídeo 4. En general, es frecuente que participe con turnos iniciativos.

15. Su uso de los gestos, la expresión facial y la comunicación no verbal, complementa y matiza su lenguaje adecuadamente pero no lo sustituye (Gestualidad natural)

Fragmento del vídeo 3 (00:10”)

- F: (...) claro, como me alegro de que Miguel sea como yo porque además me alegro um um um montón de que tú apre- aprendas muchas cosas con él. (Acaricia la mano de D)

- D: Gracias.

En numerosas ocasiones, el sujeto complementa su lenguaje oral con el uso de gestos, pero estos gestos no lo sustituyen sino que lo apoyan (ya que no presenta problemas articulatorios o léxicos que requieran una gestualidad sustitutiva).

16. Mirada. Utiliza la mirada comunicativamente, para confirmar la escucha y comprensión, para ceder o pedir el turno...

$\mathrm{F}$ dirige la mirada alternativamente hacia uno u otro interlocutor cuando desea que éste participe o se integre en la conversación. Es una manera de seleccionar el hablante siguiente, como ha puesto de manifiesto el análisis de la conversación (Sacks, Schegloff y Jefferson, 1974) y como vimos en el fragmento tomado del vídeo 4 para ilustrar el ítem 15. Es frecuente que mantenga el contacto visual en una conversación 
con interlocutores conocidos, como se puede apreciar en los vídeos 3 y 4 , y en una situación relajada de comunicación. Sin embargo, en otras situaciones, como en la entrevista inicial (vídeo 1) el contacto visual se torna esquivo, lo que puede deberse a la tensión generada por un formato de interacción asimétrico (entrevista).

17. Prioridad. Sus intervenciones son socialmente adecuadas conforme a las convenciones de cortesía y educación.

Este ítem se puede evaluar en las segundas partes de pares adyacentes, en las que F no realiza turnos de inicio. En esta muestra, lo más notorio es que $\mathrm{F}$ se adecua bien a la prioridad de dar respuestas a preguntas, tanto en la situación de entrevista (ej., fragmentos 00'00"-00'25", 00'52" y 01'49" del vídeo 1, citados arriba) como en conversación libre con iguales (fragmento 00'06”-00'20” y 02'42” del vídeo 4, también citados arriba). No hemos encontrado en la muestra oportunidades para valorar la adecuación de las segundas partes en pares más ilustrativos de normas de cortesía, como entrega-agradecimiento o petición-concesión de disculpa.

\section{Discusión}

En primer lugar, la aplicación del PREP nos permite realizar un recorrido general por las habilidades y dificultades que presenta nuestro participante. El Anexo I recoge las puntuaciones que asignamos a $\mathrm{F}$ en el PREP-INIA. Aquí detallamos algunos matices que no se recogen en el formulario:

-Las dificultades puntuales de articulación derivan en autorreparaciones conversacionales y limitan, en algunos momentos, la inteligibilidad del discurso.

-Se aprecian en ocasiones circunloquios y desviaciones de tema a medida que avanza la conversación, sobre todo cuando no domina el tema.

-La máxima de cantidad está alterada en la mayoría de las interacciones: en situaciones de poco control el sujeto aporta más información de la requerida y cuando hay un mayor control las intervenciones del sujeto se acortan. En cuanto a la máxima de manera, las intervenciones no son claras en su totalidad, pues se producen cambios de tema rápidos y descarrilamientos temáticos.

-Al construir relatos, $\mathrm{F}$ aporta información excesiva y no relacionada con la idea anterior; los cambios de tema se orientan en su mayoría hacia el deporte y se introducen bruscamente. El sujeto utiliza ciertas expresiones estereotipadas (expresiones formulaicas propias). 
-En ocasiones, el sujeto restringe la participación de los demás interlocutores.

Teniendo en cuenta las características del participante y, apoyando los resultados que se desprenden de las investigaciones realizadas por Price-Williams y Sabsay (1979) y Leudar, Fraser \& Jeeves (1981), podemos decir que la competencia comunicativa de nuestro participante es adecuada a pesar de los déficits mencionados. Con todo, los resultados que muestra $\mathrm{F}$ confirman un perfil de habilidades con puntos fuertes y débiles (Abbeduto, Warren \& Conners, 2007).

Según nuestro análisis, uno de los puntos fuertes lo constituye la pragmática interactiva. En línea con Abbeduto y Rosenberg (1980), no hemos apreciado disrupciones de la toma de turnos, y el uso de reglas conversacionales es adecuado. Los solapamientos son escasos y no perturban el intercambio (ver Peskett \& Wootton, 1985).

En cuanto a la pragmática enunciativa, F no muestra dificultades con las dimensiones locutiva e ilocutiva de los actos de habla, en línea con las observaciones de Abbeduto y Rosenberg (1980) sobre el SD.

Respecto a la pragmática textual, las dificultades que presenta el participante en relación con la coherencia y la cohesión pueden deberse a la longitud de enunciado, en línea con las observaciones de Cicchetti y Beeghley (1990), pues las intervenciones demasiado largas pueden comprometer la coherencia y la cohesión de las exposiciones si incluyen información no relevante. Este aspecto también podría afectar a las máximas de cantidad, manera y relevancia, ya que el exceso de información provoca derivaciones temáticas, afectando así a la pertinencia y adecuación de las intervenciones del sujeto en la conversación.

Así pues, nuestro análisis respalda el planteamiento general de Beeghly et al. (1990) en relación con una competencia pragmática básica en SD cercana a la normalidad, si bien este estudio se ha centrado en un caso, sin permitir comparaciones con sujetos de desarrollo típico.

En relación con el PREP-INIA, confirmamos su utilidad para el análisis de componentes pragmáticos en SD de alto rendimiento. El diseño del instrumento, configurado como un listado de cuestiones específicas, permite analizar las conductas comunicativas del sujeto y establecer conclusiones claras para la mayoría de los ítems propuestos. 


\section{Líneas futuras de investigación}

En el campo del análisis del componente pragmático en SD, sugerimos las siguientes aportaciones para la investigación futura:

-La exploración de más casos y más diversos en cuanto a rango de edad, sexo y niveles de rendimiento.

-El mantenimiento y perfeccionamiento del planteamiento propuesto aquí en cuanto al diseño y selección de tareas para la muestra de lenguaje. En esta propuesta provocamos situaciones de conversación espontánea con iguales, entrevista con profesional de referencia, sesión de logopedia, conversación en pareja y monólogo; este diseño se podría combinar y completar con otras situaciones más constreñidas que permitan evaluar todo el rango de competencia pragmática incluido en el PREP induciendo tipos de actos verbales que correspondan a dimensiones concretas.

-Ampliar la recogida de muestras de lenguaje a otros entornos donde se desenvuelve el sujeto y diseñar situaciones de comunicación para registrar intercambios con un rango más variado de interlocutores (familiares, profesionales, desconocidos, etc.) en diferentes contextos.

\section{Referencias}

Abbeduto, L. (2008). Pragmatic development. Down Syndrome Research and Practice, 07/2008, pp. 57-59. Recuperado el 15 de abril de 2013. Disponible en: <http://www.down-syndrome.org/reviews/2078/>

Abbeduto, L., y Rosenberg S. (1980). The communicative competence of mildly retarded adults. Applied Psycholinguistics, 1, 405-426.

Abbeduto, L., Davies, B. y Furman, L., (1988). The development of speech act comprehension in mentally retarded individuals and nonretarded children. Child Development, 59, 1460-1472.

Abbedutto, L., Davies, B., Solesby, S., y Furman, L. (1991). Identifying the referents of spoken messages: Use of context and clarification requests by children with and without mental retardation. American Journal on Mental Retardation, 5, 551562.

Abbeduto L., Murphy, M.M., Richmond, E., Amman, A., Beth, P., y Weissman, M.D. (2006). Collaboration in referential communication: Comparison of youth with Down syndrome or fragile X syndrome. American Journal on Mental Retardation, 111, 170-183. 
Abbeduto, L., Warren, S. F., y Conners, F. A. (2007). Language development in Down syndrome: From the prelinguistic period to the acquisition of literacy. Mental Retardation and Developmental Disabilities Research Reviews, 13, 247-261

Abbeduto, L., Murphy, M.M., Kover, S.T., Karadottir, S., Amman, A., y Bruno, L. (2008). Signaling noncomprehension of language: A comparison of fragile $X$ syndrome and Down syndrome. American Journal on Mental Retardation, 113, 214-230.

Austin, J. L (1962). How to do things with words. Oxford: Clarendon Press.

Beeghly, M., Weiss-Perry, B., y Cicchetti, D. (1990). Beyond sensorymotor functioning: Early communicative and play development of children with Down syndrome. En D. Cicchetti y M. Beeghly (eds.), Children with Down syndrome: A developmental perspective, Cambridge: Cambridge University Press, pp. 329-368.

Berglund, E., Eriksson, M., y Johansson I. (2001). Parental reports of spoken language skills in children with Down syndrome. Journal of Speech, Language, and Hearing Research, 44, 179-191.

Boudreau, D.M., y Chapman, R.S. (2000). The relationship between event representation and linguistic skill in narratives of children and adolescents with Down syndrome. Journal of Speech, Language, and Hearing Research, 43, 1146-1159.

Caselli, M., Vicari, S., Longobardi, E., Lami, L., Pizzoli, C., y Stella, G. (1998). Gestures and words in early development of children with Down Syndrome. Journal of Speech, Language, and Hearing Research, 41, 1125-1135.

Chapman, R.S., Kay-Raining Bird, E., y Schwartz, S.E. (1990). Fast mapping of words in event contexts by children with Down Syndrome. Journal of Speech and Hearing Disorders, 55, 761-770.

Chapman, R.S., Schwartz, S.E., Kay-Raining, E., y Bird, E. (1991). Language skills of children and adolescents with Down syndrome. I. Comprehension. Journal of Speech, Language, and Hearing Research, 34, 1106-1120.

Cicchetti D., y Beeghly M. (1990) Children with Down Syndrome: A Developmental Perspective. Cambridge: Cambridge University Press.

Coggings, T. E., Carpenter, R. L., y Owings N. O. (1983). Examining early intentional communication in Down's syndrome and non-retarded children. British Journal of Disorders of Communication, 18, 98-106. 
Coggins, T. E., y Stoel-Gammon, C. (1982). Clarification strategies used by four Down's Syndrome children for maintaining normal conversational interaction. Education and Training of the Mentally Retarded, 17, 65-67.

Díaz, F. (2010). Lenguaje espontáneo en el trabajo logopédico. En B. Gallardo y V. Moreno (Eds.), Estudios de lingüística clínica, V: Aplicaciones clínicas. (pp. 2948). Valencia: Publicaciones de la Universitat de Valencia.

Down, J. L. (1866). Observations on an ethnic classification of idiots. London Hospital Reports, 3, 259-262.

Gallardo-Paúls B. (2006). Más allá de las palabras y su estructura: las categorías del componente pragmático. En Garayzábal E., (Ed.), Lingüística clínica y Logopedia (pp. 81-196). Madrid: A. Machado Libros.

Gallardo, B. (2008). Pragmática Textual y TDAH. En VVAA (Eds.) ACTAS DEL XXVI Congreso Internacional AELFA. Valencia: Universidad de Valencia/Instituto INIA Neural.

Gallardo, B. (2009). Valoración del componente pragmático a partir de datos orales. Revista de Neurología, 48, S57-S61.

Greenwald, C. A., y Leonard, L. B. (1979). Communicative and sensorymotor development of Down's Syndrome children. American Journal of Mental Deficiency, 84, 296-303.

Hesketh, L.J., y Chapman, R.S. (1998). Verb use by individuals with Down Syndrome. American Journal of Mental Retardation, 103, 288-304.

Johnston F., y Stansfield J. (1997). Expressive pragmatic skills in pre-school children with and without Down's syndrome: Parental perceptions. Journal of Intellectual Disability Research, 41, 19-29.

Laws, G., y Bishop, D.V.M. (2003). A comparison of language abilities in adolescents with Down syndrome and children with specific language impairment. Journal of Speech, Language, and Hearing Research, 46, 1324-1339.

Lejeune J. (1963). Autosomal disorders. Pediatrics, 32, 326-337.

Leudar, I., Fraser, W. I., y Jeeves, M. A. (1981). Social familiarity and communication in Down syndrome. Journal of Mental Deficiency Research, 5, 133-142.

Miles S., y Chapman, R.S. (2002). Narrative content as described by individuals with Down Syndrome and typically developing children Journal of Speech, Language, and Hearing Research, 45, 175-189. 
Miller, J.F. (1988). The developmental asynchrony of language development in children with Down syndrome. En: Nadel, L. (Ed.), The psychobiology of Down syndrome. (pp. 11-39). Baltimore: Brookes.

Moraleda, E. (2011). Análisis del desarrollo morfosintáctico en personas con síndrome de Down en el periodo infantil y adolescente. Revista de Investigación en Logopedia, 2, 121-129.

Nisbet., J., Zanella, K., y Miller, J. (1984). An analysis of conversations among handicapped students and a non handicapped peers. Exceptional Children, 51, 56-162.

Owens, R.E., y Macdonald, J. D. (1982). Communicative uses of the early speech of non-delayed and Down syndrome children. American Journal of Mental Deficiency, 86, 503-510.

Peskett, R., y Wootton, A. J. (1985). Turn taking and overlap in the speech of young Down's syndrome children. Journal of Mental Deficiency Research, 29, 263273.

Price-Williams, D., y Sabsay, S. (1979). Communicative competence among severely retarded persons. Semiótica, 26, 35-63.

Roberts, J., Long, S.H., Malkin, C., Barnes, E., Skinner, M., Hennon, E.A., y Anderson, K. (2005). A comparison of phonological skills of boys with fragile $X$ syndrome and Down syndrome. Journal of Speech, Language, and Hearing Research, 48, 980-995.

Roberts, J., Martin, G., Moskowitz, L., Harris, A., Foreman, J., y Nelson, L. (2007). Discourse skills of Boys with Fragile X Syndrome in comparison to Boys with Down Syndrome. Journal of Speech, Language, and Hearing Research, 50, 475492.

Roberts, J., Price J. y Malkin C. (2007) Language and Communication development in Down syndrome. Mental Retardation and Development Disabilities Research Reviews, 13, 26-35.

Rondal, J. A. (1988). Language development in Down's syndrome: A life-span perspective. International Journal of Behavioural Development, 11, 21-36.

Rondal, J., y Comblain, A., (1996). Language in adults with Down Syndrome. Down Syndrome Research and Practice, 4, 3-14.

Rosin, M., Swift, E., y Bless, D. (1988). Communication profiles in adolescents with Down syndrome. Journal of Childhood Communication Disorders, 12, 49-64. 
Sacks, H., Schegloff, E. A., y Jefferson, G. (1974). A simplest systematics for the organization of turn-taking for conversation. Language, 50, 696-735.

Searle, J. (1969). Speech acts. Cambridge: Cambridge University Press.

Smith, L., y Von Tetzchner, S. (1986). Communicative, sensorymotor and language skills of young children with Down syndrome. American Journal of Mental Deficiency, 91, 57-66.

Wetherby, A. M., Yonclas, D. G., y Bryan, A.A. (1989). Communicative profiles of preschool children with handicaps: implications for early identification. Journal of Speech and Hearing Disorders, 54, 148-158

Wootton, A. (1990). Pointing and interaction initiation: the behaviour of young children with Down's syndrome when looking at books. Journal of Child Language, 17, 565-589. 
Anexo 1: Puntuaciones en el protocolo de evaluación pragmática "PREP-INIA"

(Gallardo et al. 2008)

\begin{tabular}{|c|c|c|c|}
\hline \multicolumn{4}{|l|}{$\begin{array}{l}\text { La conducta verbal del paciente se ajusta a las siguientes afirmaciones } \\
\text { PRAGMÁTCA ENUNCIATVA } \\
\text { Actos de habla }\end{array}$} \\
\hline $\begin{array}{l}\text { 1. Dimensiones de los actos de habla: Articula bien las palabras, de manera que las } \\
\text { emisiones son reconocibles, sin que muestre serios problemas de }\end{array}$ & & & \\
\hline 1.1. Articulación fónica, modulación sonora (Actos enunciativos) & & ㅁ. & \\
\hline 1.2. Acceso léxico (Actos proposicionales) & $\mathbf{n}$ & & \\
\hline 1.3. Manejo de las panisas y silencios & : & & \\
\hline $\begin{array}{l}\text { 2. Las emisiones que utiliza demuestran una comprensión suficiente de lo que se le dice, } \\
\text { y son las adecuadas para conseguir su propósito commicativo, aunque puedan tener } \\
\text { fallos gramaticales o léxicos (Actos ilocutivos) }\end{array}$ & - & & \\
\hline \multicolumn{4}{|l|}{ Tareas de edición } \\
\hline $\begin{array}{l}\text { 3. Pese a tener problemas de léxico o articulación, consigue transmitir su intención } \\
\text { commicativa (ilocutividad) con: } \\
3.1 \text {. Exclamaciones, entonaciones marcadas, silabas sueltas, o palabras de } \\
\text { significado impreciso, "yyy...", "pueees", "claro"... (Actos locutivos/enunciativos) }\end{array}$ & - & & \\
\hline 3.2. Gestos y actos no verbales & $=$ & & \\
\hline 3.3. Circunloquios, rodeos, preguintas del tipo de "¿eh?, ¿no?" & E & & \\
\hline $\begin{array}{l}\text { 4. Muestra conciencia de su propia dificultad, iniciando el mismo rectificaciones en los } \\
\text { puntos problemáticos (Auto-rectificaciones conversacionales) }\end{array}$ & = & & \\
\hline \multicolumn{4}{|l|}{ Inferencias } \\
\hline $\begin{array}{l}\text { 5. Al plantearle emisiones que contienen frases hechas, refranes, etc. de sentido } \\
\text { figurado, demuestra haberlas entendido correctamente; o las usa èl mismo (Inferencias } \\
\text { trópicas lexicalizadas). }\end{array}$ & & & = \\
\hline $\begin{array}{l}\text { 6. Su conversación (pese a posibles fallos léxicos o articulatorios) avanza con relativa } \\
\text { fluidez, respetando las leyes conversacionales de cooperación que exigen proporcionar } \\
\text { información (Implicaturas conversacionales): } \\
\text { 6.1. Veraz (Máxima de la cualidad) }\end{array}$ & : & & \\
\hline 6.2. Sufficiente, ni escasa ni excesiva (Máxima de la cantidad) & & 口 & \\
\hline 6.3. Clara, concreta (Máxima de la manera) & & $\mathbf{\square}$ & \\
\hline 6.4. Relevante (Mảxima de la pertinencia) & & n & \\
\hline 6.5. En ocasiones, comprendida a partir de sentidos figurados (Impl. anómalas) & & & : \\
\hline
\end{tabular}

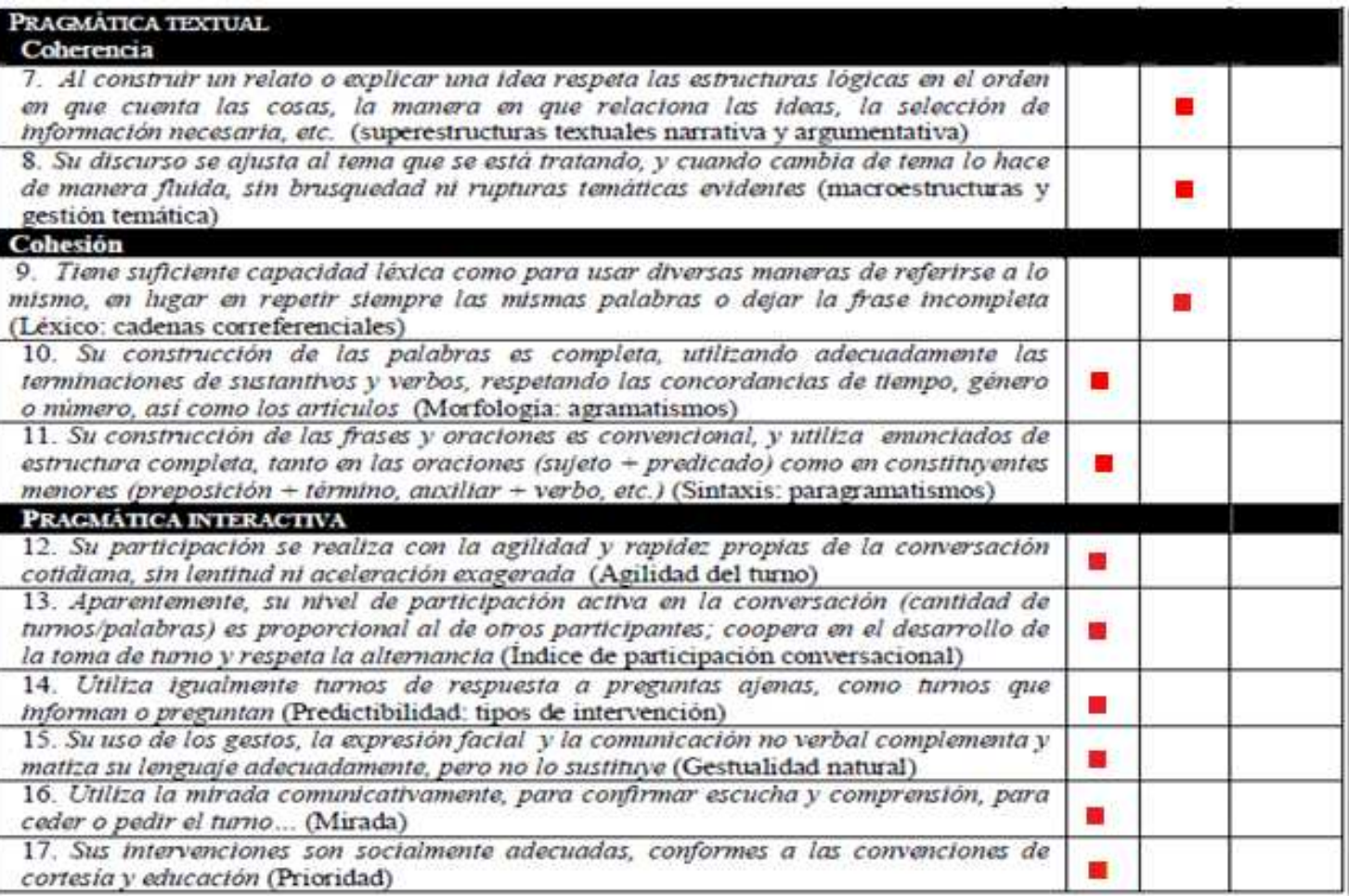


Anexo 2. Convenciones de transcripción

\begin{tabular}{|c|c|}
\hline Símbolo empleado & Valor \\
\hline Eee & Alargamiento de vocalización \\
\hline- & Autointerrupción \\
\hline / & Pausa inferior a un segundo \\
\hline$(2)$ & $\begin{array}{l}\text { Tiempo en segundos de pausa superior a un } \\
\text { segundo }\end{array}$ \\
\hline (mira hacia V) & Actos no verbales relevantes al análisis \\
\hline , . ? & $\begin{array}{l}\text { Su significado en lenguaje escrito (añadido } \\
\text { para aportar sentido gramatical a los } \\
\text { enunciados y facilitar la legibilidad de los } \\
\text { fragmentos) }\end{array}$ \\
\hline$(\ldots)$ & Habla circundante omitida del fragmento \\
\hline
\end{tabular}

